\title{
Correlação entre a amônia e o potencial evocado relacionado a eventos (P300) em pacientes cirróticos
}

\author{
Correlation between ammonia and event-related evoked potentials (P300) in cirrhotic patients
}

\section{Maurício Augusto Bragagnolo Júnior'1, Vinícius Teodoro², Lígia Mendonça Lucchesi ${ }^{3}$, Ruth Ferreira Santos ${ }^{4}$, Ana Cristina de Castro Amaral Feldner ${ }^{5}$, Tarsila Campanha da Rocha Ribeiro', Sérgio Tufik ${ }^{6}$, Mário Kondo ${ }^{7}$}

\section{RESUMO}

A amônia é a neurotoxina que melhor explica alterações cognitivas em cirróticos e o potencial evocado relacionado a eventos auditivos (P300) tem sido considerado um bom método para rastreálas. Entretanto, é ainda desconhecido se pode ele pode ser influenciado pelas concentrações de amônia. Objetivo. avaliar a correlação entre amônia arterial e o P300 em cirróticos. Método. Cirróticos sem encefalopatia hepática foram submetidos à determinação da dosagem arterial de amônia arterial, P300 e avaliação da gravidade da doença hepática. O P300 foi anormal quando a latência de P300 foi superior ao limite superior da normalidade determinado por estudo normativo. Resultados. Avaliados 48 pacientes, com P300 anormal em 36 (75\%). Aqueles com P300 anormal apresentaram amônia arterial significativamente maior do que aqueles com P300 normal (197 \pm 156 vs. $90 \pm 82 \mathrm{mmol} / \mathrm{L} ; \mathrm{p}<0,05)$. Amônia $300 \mathrm{mmol} / \mathrm{L}$ também se associou à anormalidade do $\mathrm{P} 300$. Conclusão. A amônia arterial foi significativamente maior em cirróticos com P300 anormal e maiores níveis se associaram à anormalidade do exame, sugerindo que o P300 é boa ferramenta para rastrear anormalidades cognitivas em cirróticos, desde que se correlaciona com a principal substância envolvida na fisiopatogenia da encefalopatia hepática.

Unitermos. Cirrose Hepática, Encefalopatia Hepática, Potencial Evocado P300, Cognição, Amônia.

Citação. Bragagnolo Júnior MA, Teodoro V, Lucchesi LM, Santos RF, Feldner ACCA, Ribeiro TCR, Tufik S, Kondo M. Correlação entre a amônia e o potencial evocado relacionado a eventos (P300) em pacientes cirróticos.

Trabalho realizado na Disciplina de Gastroenterologia e Departamento de Psicobiologia da UNIFESP, São Paulo-SP, Brasil

1. Gastroenterologista, Mestre, Pós-graduando da Disciplina de Gastroenterologia da Universidade Federal de São Paulo-UNIFESP, São Paulo-SP,Brasil.

2. Neurologista, Pós-graduando do Departamento de Psicobiologia da UNIFESP, São Paulo-SP, Brasil.

3. Neurofisiologista, Doutora, Médica do Departamento de Psicobiologia da UNIFESP, São Paulo-SP,Brasil.

4. Neuropsicóloga, Doutora, Psicóloga do Departamento de Psicobiologia da UNIFESP, São Paulo-SP,Brasil.

5. Gastroenterologista, Pós-graduanda da Disciplina de Gastroenterologia da UNIFESP, São Paulo-SP,Brasil.

6. Neurofisiologista, Professor Livre-Docente do Departamento de Psicobiologia da UNIFESP, São Paulo-SP,Brasil.

7. Gastroenterologista, Doutor, Professor Adjunto da Disciplina de Gastroenterologia da UNIFESP, São Paulo-SP,Brasil.

\section{SUMMARY}

Ammonia is the neurotoxin that best explains cognitive disorders in cirrhotic patients and auditory evoked-related potential (P300) can be considered a good method for these alterations screening. Although, ammonia blood concentration induced alterations on P300 are yet unknown. Objective. To evaluate correlation between arterial ammonia blood levels and P300 in cirrhotics. Method. Cirrhotics without overt hepatic encephalopathy were evaluated, including arterial ammonia blood levels determination, P300 and assessment of liver disease severity. P300 was abnormal when P300 wave latency was superior to the upper limit of normality, according a normative study. Results. 48 were evaluated and P300 abnormality was detected in 36 (75\%). P300 abnormality was associated with significant higher ammonia levels than P300 normality (197 \pm 156 vs. $90 \pm 82$ $\mathrm{mmol} / \mathrm{L}$; p<0.05). Ammonia levels $300 \mathrm{mmol} / \mathrm{L}$ were associated with P300 abnormality too. Conclusion. Arterial ammonia blood levels were significantly higher in cirrhotics with P300 abnormality as well as P300 abnormality was associated with higher ammonia levels. It suggests that P300 plays a major role in cirrhotic cognitive abnormalities screening, since it correlates with the main substance involved in hepatic encephalopathy physiopathology.

Keywords. Liver Cirrhosis, Hepatic Encephalopathy, Evoked Potentials, Event-related Eoked Potential P300, Cognition, Ammonia.

Citação. Bragagnolo Júnior MA, Teodoro V, Lucchesi LM, Santos RF, Feldner ACCA, Ribeiro TCR, Tufik S, Kondo M. Correlation between ammonia and event-related evoked potentials (P300) in cirrhotic patients.

Endereço para correspondência Maurício A Bragagnolo Júnior R. Clóvis S Motta, 138 CEP 36031-280, Juiz de Fora-MG, Brasil. E-mail: mauricio.jr@acessa.com / mab2@acessa.com

Artigo Original Recebido em: 04/02/2009 Revisado em: 05/02/2009 a 13/04/2009 Aceito em: 14/04/2009 Conflito de interesses: não 


\section{INTRODUÇÃO}

A encefalopatia hepática pode ser conceituada como um conjunto de alterações neuropsiquiátricas que ocorrem em indivíduos com insuficiência hepática e/ou hipertensão portal ${ }^{1}$. Sua fisiopatologia é complexa, possivelmente multifatorial e ainda não foi completamente compreendida a despeito das investigações realizadas nos últimos 50 anos. Várias neurotoxinas já foram aventadas como possíveis agentes causais envolvidos, mas principalmente a amônia. Esta substância, que ganha a circulação entero-hepática após ser absorvida no trato gastrointestinal, parece não ser adequadamente depurada em pacientes com cirrose hepática, causando alterações da neurotransmissão glutamatérgica e conseqüente sintomatologia neuropsiquiátrica ${ }^{2}$. A associação entre hiperamonemia e alterações neurológicas já existe há séculos, desde que estudos preliminares demonstraram o efeito da administração de carne através de fístulas de Eck em cães com anastomose porto-cava ${ }^{3}$. Já foram descritas alterações neurológicas relacionadas à elevação dos níveis de amônia em portadores de cirrose hepática após a ingestão de proteínas, sal de amônia ou resinas de troca iônica potássio-amônia ${ }^{4}$. O torpor episódico e reversível já foi documentado em um paciente submetido à derivação porto-cava para o tratamento de câncer pancreático $^{5}$. Desde as descrições iniciais até o presente momento, a amônia ainda é a candidata que melhor explica as alterações clínicas, patológicas e neuroquímicas observadas na encefalopatia hepática ${ }^{6}$.

O emprego do potencial evocado relacionado a eventos auditivos P300 tem sido utilizado em diversos estudos para avaliar enfermidades que cursam com alterações cognitivas, entre elas a encefalopatia hepática ${ }^{7-11}$ e vem sendo descrito como um dos métodos mais sensíveis para seu diagnóstico, inclusive em formas ainda subclínicas. $\mathrm{O}$ achado mais caracteristicamente observado nestes indivíduos é o retardo na latência da onda $\mathrm{P} 300$ em comparação a controles saudáveis. Pouco se sabe, no entanto, se este fenômeno neurofisiológico está relacionado à elevação da concentração sérica de amônia em pacientes com cirrose hepática, levando em conta que a hiperamonemia tem sido considerada o fator mais importante na gênese da encefalopatia hepática. $\mathrm{O}$ presente estudo tem por objetivo estudar a possível correlação entre a concentração sérica de amônia e as alterações detectadas no P300 de indivíduos cirróticos, desde que ambos são considerados eventos associados à presença de alterações neuropsiquiátricas e disfunções cognitivas neste grupo.

\section{MÉTODO \\ Amostra}

Foram avaliados cirróticos provenientes do ambulatório de hepatologia da Universidade Federal de São Paulo (UNIFESP), com investigação etiológica e estratificação da gravidade da doença pela classificação de Child-Pugh em todos ${ }^{12}$. Foram excluídos aqueles com encefalopatia hepática ao exame clínico inicial, aqueles que apresentaram sangramento digestivo no último mês ou insuficiência renal (creatinina sérica $>2,0 \mathrm{mg} / \mathrm{dL}$ ), diagnóstico de carcinoma hepatocelular, uso de medicação psicoativa ou lactulose nas últimas 2 semanas, uso de álcool nos últimos 6 meses, idade inferior a 18 ou superior a 65 anos e recusa a participar do estudo. Aqueles que concordaram em participar do estudo assinaram o Termo de Consentimento Livre e Informado aprovado pela Comissão de Ética em Pesquisa da Unifesp (1609/06).

\section{Procedimento}

A dosagem de amônia foi feita por método colorimétrico azul de bromofenol (Vitros Chemistry Products: valor de referência para adultos da dosagem arterial $=9-33 \mathrm{mmol} / \mathrm{L}$ ), através da coleta de sangue em artéria radial ou braquial em seringa heparinizada.

A avaliação do P300 foi feita com aparelho NIHON KONDEN, NEUROPACK, MEB5508K, com eletrodos ativos nas posições frontal, central e parietal da linha mediana, respectivamente FZ, CZ e PZ, de acordo com o sistema internacional $10-20^{13}$. Eletrodos de referência foram colocados em A1 e A2, ligados entre si. O eletrodo "terra" foi posicionado em FPZ. Foram também colocados eletrodos acima da sobrancelha direita e no canto externo do olho esquerdo para controle do eletrooculograma. A impedância elétrica foi mantida abaixo de $10 \mathrm{KW}$. O paradigma utilizado foi o "oddball"14, com estímulos auditivos apresentados bilateralmente através de fones de ouvido com tons de $70 \mathrm{~dB}$ com $10 \mathrm{~ms}$, em "subida / descida" e "plateau" de 100 ms. Dois tons foram apresentados de forma aleatória: estímulo raro, de $1500 \mathrm{~Hz}$ (com 20\% de probabilidade de aparecimento) e estímulo freqüente, de $800 \mathrm{~Hz}$ (com $80 \%$ de probabilidade). O intervalo entre os estímulos foi fixado em 2 segundos. A latência da 
onda P300 foi dada pela medida da latência entre o estímulo dado e o surgimento da primeira e maior onda positiva registrada entre $250-500 \mathrm{~ms}$ do estímulo raro. Para estimar o limite superior da normalidade para a latência da onda P300 aferida em CZ para determinada idade foi utilizada a fórmula $\mathrm{x}=(2,23 \mathrm{x}$ idade) +234 . Esta fórmula foi definida em um estudo normativo do P300 para controles saudáveis estudados previamente em nossa instituição, por Luccas et al. ${ }^{15}$. Foram considerados anormais os testes onde a latência da onda P300 foi superior ao limite superior da normalidade calculado pela fórmula.

\section{Análise Estatística}

A análise estatística foi feita pelo programa Statistica, versão 5.0 (edição 97), comparando as variáveis quantitativas pelo teste $\mathrm{t}$ de Student ou análise de variância, e as variáveis qualitativas pelo teste qui-quadrado, com correção de Yates. O nível de significância adotado foi o de $5 \%$.

\section{RESULTADOS}

Foram avaliados 48 pacientes com cirrose

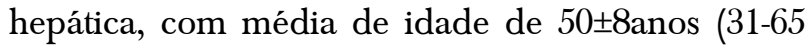
anos), sendo $38(79 \%)$ do sexo masculino. As etiologias mais freqüentes foram: álcool em 21 (44\%) e vírus $\mathrm{C}$ em 12 (25\%). Vinte e um (44\%) eram Child A, 18 (37\%) Child B e 9 (19\%) Child C, com média de $7,3 \pm 2,1$ pontos na referida classificação (5-12 pontos). A amônia foi dosada em 43 pacientes, sendo a média de $172 \pm 149 \mathrm{mmol} / \mathrm{L}(33-543 \mathrm{mmol} / \mathrm{L})$ - Tabela 1.

Tabela 1. Características gerais do grupo estudado.

\begin{tabular}{|l|l|}
\hline Caracteristicas & Valores \\
\hline Idade (anos) & $50 \pm 8$ \\
\hline Sexo Masculino & $38(79 \%)$ \\
\hline Etiologia & \\
Álcool & $21(44 \%)$ \\
Virus C & $12(25 \%)$ \\
Álcool + Vírus C & $08(17 \%)$ \\
Vírus B & $02(04 \%)$ \\
Álcool + Vírus B & $01(02 \%)$ \\
Criptogênica & $04(08 \%)$ \\
\hline Child-Pugh (pontos) & $7,3 \pm 2,1$ \\
Child A & $21(44 \%)$ \\
Child B & $18(37 \%)$ \\
Child C & $09(19 \%)$ \\
\hline Amônia arterial (mmol/L) & $172 \pm 149$ \\
\hline
\end{tabular}

*Valores apresentados em média \pm desvio padrão e quantidade de pessoas (percentual)

Considerando os valores de referência para as concentrações arteriais de amônia de adultos $(9-33 \mathrm{mmol} / \mathrm{L})$, apenas um paciente obteve normalidade no exame, ou seja, hiperamonemia foi observada em $98 \%$ dos casos de cirrose hepática, mesmo sem encefalopatia hepática.

As concentrações arteriais de amônia não foram estatisticamente diferentes nas classes A, B e C de Child-Pugh (Tabela 2). Observou-se latência da onda P300 de $365,4 \pm 42,16 \mathrm{~ms}$ (282-496ms), sendo que 36 (75\%) obtiveram retardo, ou seja, P300 anormal. A gravidade da cirrose avaliada pela classificação de Child-Pugh não interferiu significativamente na latência de P300 (Child A=359 $\pm 31 \mathrm{~ms}$, Child $\mathrm{B}=364 \pm 53 \mathrm{~ms}$; Child $\mathrm{C}=383 \pm 40 \mathrm{~ms} ; \mathrm{p}=0,35$; análise de variância), Gráfico 1. Embora a correlação entre a concentração arterial de amônia e o valor da latência da onda P300 tenha sido fraca e sem significado estatístico (Correlação de Pearson: $r=0,11 ; \mathrm{p}>0,05)$, observou-se concentrações arteriais de amônia significativamente maiores naqueles com P300 anormal quando comparados àqueles com $\mathrm{P} 300$ normal $(197 \pm 156 \mathrm{mmol} / \mathrm{L}$ vs. $90 \pm 82 \mathrm{mmol} / \mathrm{L} ; \mathrm{p}<0,05)$, Gráfico 2. De forma semelhante, pacientes com amônia $300 \mathrm{mmol} / \mathrm{L}$ também apresentaram anormalidade do P300 mais frequentemente do que aqueles com amônia arterial $<300 \mathrm{mmol} / \mathrm{L}(100 \%$ vs. $68 \%$, respectivamente; $\mathrm{p}<0,05$ - qui-quadrado com correção de Yates).

Tabela 2. Concentrações arteriais de amônia nas diferentes classes de Child=Pugh

\begin{tabular}{|l|c|c|}
\hline & $\mathrm{n}$ & Amônia $(\mathrm{mmol} / \mathrm{L})=$ média $\pm \mathrm{DP}$ \\
\hline Child A & 19 & $133 \pm 132$ \\
\hline Child B & 16 & $236 \pm 169$ \\
\hline Child C & 08 & $136 \pm 112$ \\
\hline Todas & 43 & $172 \pm 149$ \\
\hline
\end{tabular}

\section{DISCUSSÃO}

A amônia ainda é a principal neurotoxina associada às alterações clínicas, patológicas e neuroquímicas da encefalopatia hepática6. A freqüência de hiperamonemia na amostra estudada foi de 98\%. De forma semelhante, um estudo clássico também demonstrou que o teste arterial de tolerância à amônia, de semelhante significado à presença de hiperamonemia arterial, foi positivo em $97,6 \%$ cirróticos sem encefalopatia hepática ${ }^{16}$. Embora seja reconhecido o papel da amônia na fisiopatogenia da encefalopatia hepática, este estudo também reafirma o papel limitado que tem para seu diagnóstico, des- 
de que quase invariavelmente encontra-se elevada em indivíduos com cirrose hepática, mesmo aqueles sem encefalopatia hepática ${ }^{17}$.

Supõe-se que a dosagem arterial da amônia seja mais fidedigna que a venosa, considerando a eliminação de fatores como a captação periférica de amônia pelos músculos e porque refletem de forma mais acurada os níveis de amônia que atingem a circulação cerebral ${ }^{18,19}$, sendo, portanto, a empregada neste estudo. Embora já tenha sido demonstrado previamente que os níveis arteriais de amônia se correlacionam com o grau de encefalopatia hepática, pouco se conhece sobre sua correlação com a gravidade da doença hepática. No presente estudo, indivíduos das classes A, B e C de ChildPugh não apresentaram níveis arteriais de amônia ou latência de P300 significativamente diferentes entre si. Da mesma forma, também não encontraram correlação significativa entre a latência de P300 e a gravidade da cirrose hepática avaliada pela classificação de Child-Pugh ${ }^{20}$. Embora ainda não descrita a correlação entre a gravidade da cirrose hepática e as concentrações arteriais de amônia, presume-se, por dedução, que também seja de pouca relevância esta correlação, desde não encontraram influência significativa da gravidade da cirrose hepática sobre a presença de encefalopatia hepática subclínica em cirróticos $^{21}$, condição sabidamente associada à hiperamonemia ${ }^{22}$.
Até o momento, não existe um exame "padrão-ouro" para o rastreamento de anormalidades cognitivas em pacientes cirróticos com ou sem encefalopatia hepática. Embora os mais utilizados sejam os testes neuropsicológicos e os testes neurofisiológicos (como o P300), ambos são aplicáveis a este propósito segundo recomendações consensuais ${ }^{1}$. No entanto, o P300 tem sido apontado por alguns autores como a melhor ferramenta para a detecção destas anormalidades, desde que vem se mostrando mais sensível do que os testes neuropsicológicos ${ }^{8,23}$. Embora haja a necessidade de equipamento específico e nem sempre disponível em serviços de hepatologia, também tem se destacado por não sofrer influência do aprendizado e do grau de escolaridade, ao contrário dos testes neuropsicológicos ${ }^{24}$. No presente estudo, o P300 esteve alterado em 75\% dos cirróticos, à semelhança dos resultados relatados previamente na literatura ${ }^{25}$. As concentrações arteriais de amônia foram significativamente maiores naqueles com P300 anormal quando comparados àqueles com P300 normal $(\mathrm{p}<0,05)$. De forma semelhante, pacientes com amônia arterial $300 \mathrm{mmol} / \mathrm{L}$ apresentaram P300 mais frequentemente alterado do que aqueles com amônia arterial $<300 \mathrm{mmol} / \mathrm{L}(\mathrm{p}<0,05)$.

Gráfico 1. Latência da onda P300 nas diferentes classes de Child-Pugh.

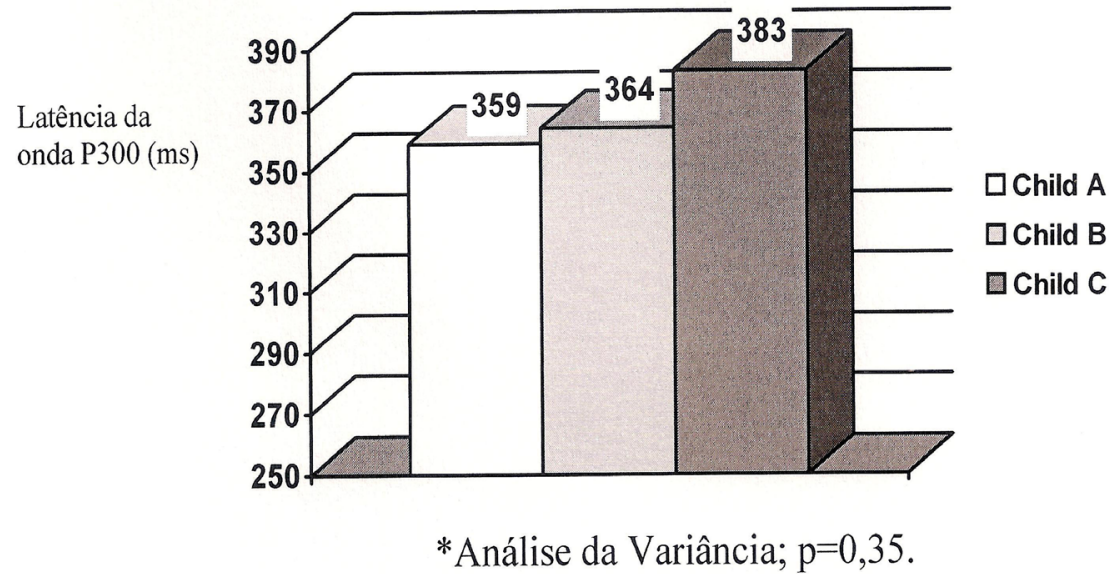


Gráfico 2 - Comparação da amônia arterial em cirróticos

com P300 normal e anormal

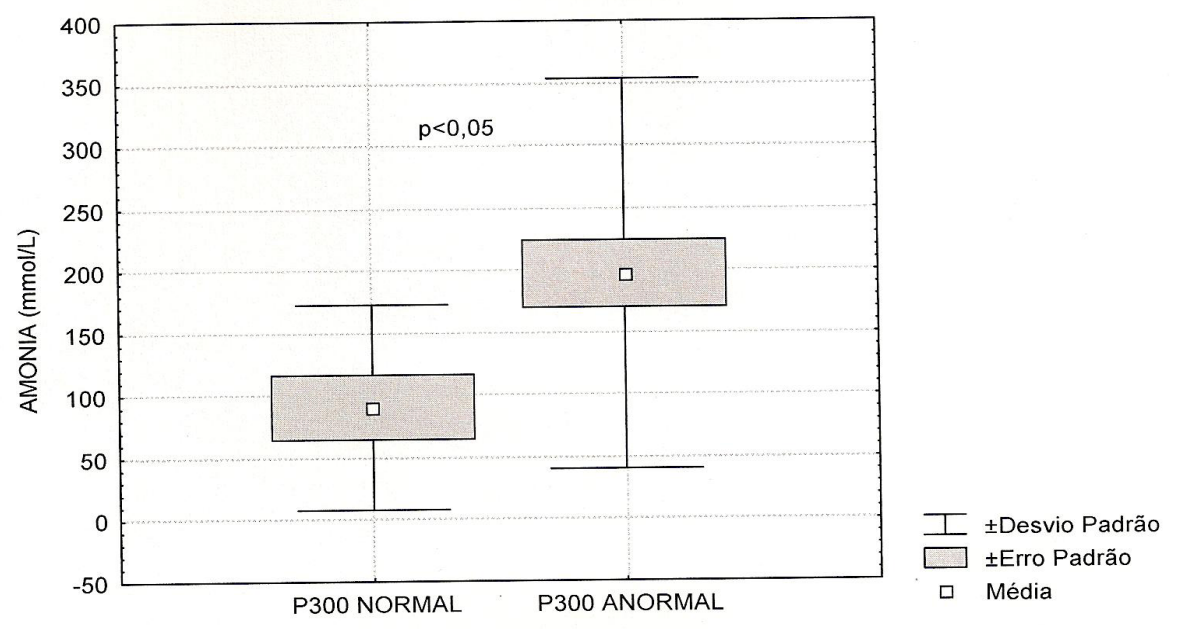

Cabe ressaltar que este achado infere na associação entre a amônia arterial e P300, o que poderia ser mais um dado falando a favor da superioridade deste exame em relação aos testes neuropsicológicos para a detecção de alterações cognitivas em cirróticos, desde que o seu resultado sofre influência da principal substância envolvida na fisiopatogenia da encefalopatia hepática: a amônia.

Indo um pouco além, e tentando explicar o motivo da associação entre a hiperamonemia e os resultados do P300, vale lembrar que é bem conhecida influência da cirrose hepática e da hiperamonemia sobre a neurotransmissão glutamatérgica. $\mathrm{O}$ aumento da concentração extracelular de glutamato cerebral resultante de uma maior depuração local de amônia é, pelo menos em parte, responsável pela disfunção cognitiva observada em indivíduos com encefalopatia hepática. Estudos experimentais prévios já demonstraram um aumento da concentração de glutamato extracelular no hipocampo de modelos animais com encefalopatia hepática e hiperamonemia $^{26,27}$. Se considerarmos que a onda P300 é sabidamente gerada no lobo temporal medial, incluindo o hipocampo ${ }^{28,29}$, pode-se supor que hiperamonemia poderia induzir alterações na neurotransmissão glutamatérgica desta região cerebral, podendo induzir um retardo na latência da onda P300 e a anormalidade do exame P300 observada em nossos pacientes. Cabe, no entanto, mais estudos prospectivos no sentido de confirmar tal suposição.

\section{CONCLUSÃO}

Cirróticos sem encefalopatia hepática e P300 anormal apresentaram concentrações arteriais de amônia significativamente maiores do que aqueles com P300 normal, da mesma forma que concentrações de amônia $300 \mathrm{mmol} / \mathrm{L}$ estão associadas à maior freqüência de anormalidade no referido exame. Estes achados permitem inferir que o P300 é um exame interessante para a pesquisa de encefalopatia hepática e anormalidades cognitivas em pacientes com cirrose, desde que se correlaciona com a principal substância envolvida na fisiopatogenia desta condição.

\section{REFERÊNCIAS BIBLIOGRÁFICAS}

1.Ferenci P, Lockwood A. Hepatic Encephalopathy - Definition, nomenclature, diagnosis, and quantification: Final report of the Working Party at the 11th World Congress of Gastroenterology, Vienna, 1998. Hepatology 2002;35:716-21.

2.Albrecht J, Jones EA. Hepatic encephalopathy: molecular mechanisms underlying the clinical syndrome. J Neurol Sci 1999;170:138-46.

3.Hahn M, Massen O, Nencki M, Pavlov J. Die Eck-sche Fistel zwiischen der unteren Hohlvene um der Pfortader und ihre Folgen für den Organismus. Arch Exp Path Pharmakol 1893;32:161.

4.Phillips GB, Schwartz R, Gabuzda GJ, Davidson CS. The syndrom of impending hepatic coma in patients with cirrhosis of the liver given certain nitrogenous substances. New Engl J Med 1952;247:239.

5.Mcdermott WV, Adams AD. Episodic stupor associated with an eck fistula in the human with particular reference to the metabolism of ammonia. J Clin Invest. 1953;33:1-9.

6.Norenberg MD. Astrocytic-ammonia interactions in hepatic encephalopathy. Semin Liver Dis 1996;16:245-52.

7.Davies MG, Rowan MJ, Macmathuna P, Keeling PWN, Weir DG, Feely J. The auditory P300 event-related potential: an objective marker of the encephalopathy of chronic liver disease. Hepatology 1990;12:688-94. 
8.Kullmann F, Hollerbach S, Holstege A, Scholmerich J. Subclinical hepatic encephalopathy: the diagnostic value of evoked potentials. J Hepatol 1995;22:101-10

9.Saxena N, Bhatia M, Joshi YK, Garg PK, Tandon RK. Utility of P300 auditory event related potential in detecting cognitive dysfunction in patients with cirrhosis of the liver. Neurol India 2001;49:350-4.

10.Romero-Gómez M, Boza F, García-Valdecasas MS, García E, AguilarReina J. Subclinical hepatic encephalopathy predicts the development of overt hepatic encephalopathy. Am J Gastroenterol 2001;96:2718-23.

11.Saxena N, Bhatia M, Joshi YK, Garg PK, Dwivedi SN, Tandon RK. Electrophysiological and neuropsychological tests for the diagnosis of subclinical hepatic encephalopathy and prediction of overt encephalopathy. Liver 2002;22:190-7.

12.Pugh RNH, Murray-Lion IM, Dawson JL, Pietroni MC, Williams R. Transection of the oesophagus for bleeding oesophageal varices. $\mathrm{Br} \mathrm{J}$ Surg 1973;60:646-9.

13.Jasper HH. Report of the comitee on methods of clinical examination in electroencephalography. Electroencephalogr Clin Neurophisiol 1958;10:370-5.

14.Polich J. P300 in clinical applications: meaning, method, and measurement in electroencephalography: basic principles, clinical applications, and related fields. 3rd ed. Baltimore: Williams \& Wilkins, 1993, 1005p. 15.Luccas FJC, Lucchesi LM, Lopes JA. Potenciais evocados ligados a eventos P300: estudo normativo. In: 13 Congresso Brasileiro de Neurologia 1988, São Paulo. Anais do Congresso 1988, 33p.

16.Stahl J. Studies of the blood ammonia in liver disease. Ann Intern Med. 1963;58:1-24.

17.Nicolao F, Efrati C, Masini A, Merli M, Attili AF, Riggio O. Role of determination of partial pressure of ammonia in cirrhotic patients with and without hepatic encephalopathy. J Hepatol 2003;38:441-6.

18.Pappas SC, Jones EA. Methods for assessing hepatic encephalopathy. Semin Liver Dis 1983;3:298-307.

19.Bessman SP, Bradley JE. Uptake of ammonia by muscle. N Engl J Med 1955;253:1143-7.
20.Hollerbach S, Kullmann F, Rudiger F, Lock G, Geissler A, Wimmer $\mathrm{M}$, et al. Auditory event-related cerebral potentials (P300) in hepatic encephalopathy - topographic distribution and correlation wiyh clinical and psychometric assessment. Hepatogastroenterol 1997;44:1002-12. 21.Jover R, Company L, Gutierrez A, Zapater P, Perez-Serra J, Girona $\mathrm{E}$, et al. Minimal hepatic encephalopathy and extrapyramidal signs in patients with cirrhosis. Am J Gastroenterol 2003;98:1599-604.

22.Marchesini G, Zoli M, Dondi C. Prevalence of subclinical hepatic encephalopathy in cirrhosis and relationship to plasma aminoacid balance. Dig Dis Sci 1980;25:763-8.

23.Yang SS, Wu CH, Chiang TR, Chen DS. Somatosensory evoked potentials in subclinical portosystemic encephalopathy: a comparison with psychometric tests. Hepatol 1998;27:357-61.

24.Ortiz M, Jacas C, Cordoba J. Minimal hepatic encephalopathy: diagnosis, clinical significance and recommendations. J Hepatol 2005;42(suppl1):S45-53.

25.Kugler CFA, Lotterer E, Petter J, Wensing G, Taghavy A, Hahn EG, et al. Visual event-related P300 potentials in early portosystemic encephalopathy. Gastroenterol 1992;103:302-10.

26.Hamberger A, Nyström B. Extra and intracellular amino acids in the hippocampus during development of hepatic encephalopathy. Neurochem Res 1984;9:1181-92.

27.McArdle P, Penning DH, Dexter F, Reynolds JD. Flumazenil does not affect the increase in rat hippocampal extracellular glutamate concentration produced during thioacetamide-induced hepatic encephalopathy. Metab Brain Dis 1996;11:329-42.

28.Halgren E, Squires N, Wilson C, Rohrbaugh J, Bab T, Crandall P. Endogenous potentials in the human hippocampal formation and amygdala by infrequent events. Science 1980;210:803-5.

29.Mccarthy G, Wood CC, Williamson PD, Spencer DD. Task-dependent field potentials in human hippocampal formation. J Neurosci $1989 ; 9: 4253-68$ 